\title{
Fluid Absorption Alteration
}

National Cancer Institute

\section{Source}

National Cancer Institute. Fluid Absorption Alteration. NCI Thesaurus. Code C41547.

A finding indicating a deficiency or excess in absorption of one or more fluids. 